Click www.researchjournal.co.in/online/subdetail.html to purchase.

I J

DOI: 10.15740/HAS/IJPS/11.2/359-363

Visit us - www.researchjournal.co.in

\title{
ReSEARChArticle
}

\section{Response of manures and industrial by-products for cane yield and post harvest NPK status}

\author{
D. VENKATAKRISHNAN
}

\section{SUMMARY}

Field experiment was conducted in clay loam soil at Periyanellicollai village at Chidambaram taluka, Cuddalore district, Tamil Nadu. The soil of Periyanellikollai was classified as Typic Haplustert comes under Kondal series having sandy loam texture. The available nutrient status was low in $\mathrm{N}$, medium in $\mathrm{P}$ and $\mathrm{K}$. The treatments considered of $\mathrm{T}_{1}-$ Seasoned pressmud @25 t ha ${ }^{-1}, \mathrm{~T}_{2}-\mathrm{T}_{1}+$ Enriched gypsum @ $1 \mathrm{t} \mathrm{ha}^{-1}, \mathrm{~T}_{3}-\mathrm{T}_{2}+\mathrm{ZnSO}_{4} @ 37.5 \mathrm{~kg} \mathrm{ha}^{-1}, \mathrm{~T}_{4}-\mathrm{T}_{1}+$ Lignite fly ash @ $25 \mathrm{tha}^{-1}, \mathrm{~T}_{5}-\mathrm{T}_{1}+$ Vermicompost @ $5 \mathrm{tha}^{-1}, \mathrm{~T}_{6}-$ Vermicompost @ $5 \mathrm{tha}^{-1}+$ enriched gypsum @ $1 \mathrm{tha}^{-1}, \mathrm{~T}_{7}-\mathrm{T}_{6}+\mathrm{ZnSO}_{4}$ @ $37.5 \mathrm{~kg} \mathrm{ha}^{-1}, \mathrm{~T}_{8}-$ Vermicompost @ $5 \mathrm{t} \mathrm{ha}^{-1}+$ lignite fly ash @ $25 \mathrm{tha}^{-1}, \mathrm{~T}_{9}$ - Biocompost @ $5 \mathrm{tha}^{-1}, \mathrm{~T}_{10}-\mathrm{T}_{9}+$ Enriched gypsum @ $1 \mathrm{tha}^{-1}, \mathrm{~T}_{11}-\mathrm{T}_{10}+\mathrm{ZnSO}_{4} @ 37.5 \mathrm{~kg} \mathrm{ha}^{-1}, \mathrm{~T}_{12}-\mathrm{T}_{9}+$ Lignite fly ash @ $25 \mathrm{t} \mathrm{ha}^{-1}, \mathrm{~T}_{13}-\mathrm{FYM} @ 10 \mathrm{tha}^{-1}, \mathrm{~T}_{14}-$ recommended dose of fertilizer. All the plots were applied with recommended of dose of fertilizers 275:62.5:150 of $\mathrm{N}, \mathrm{P}_{2} \mathrm{O}_{5}$ and $\mathrm{K}_{2} \mathrm{O} \mathrm{kg} \mathrm{ha}{ }^{-1}$. The highest cane yield of $169.74 \mathrm{tha}^{-1}$ was obtained with $\mathrm{T}_{3}$ received seasoned pressmud @ 25 $\mathrm{t} \mathrm{ha}^{-1}+$ enriched gypsum @ $1 \mathrm{t} \mathrm{ha}{ }^{-1}+\mathrm{ZnSO}_{4} @ 37.5 \mathrm{~kg} \mathrm{ha}^{-1}$. The highest content of post harvest nitrogen $\left(278 \mathrm{~kg} \mathrm{ha}^{-1}\right)$ and post harvest phosphorus $\left(19.3 \mathrm{~kg} \mathrm{ha}^{-1}\right)$ was maximum $\mathrm{T}_{3}$ receiving seasoned pressmud @ $25 \mathrm{tha}^{-1}+$ enriched gypsum @ $1 \mathrm{t} \mathrm{ha}^{-1}+\mathrm{ZnSO}_{4} @ 37.5 \mathrm{~kg} \mathrm{ha}^{-1}$. The post harvest available potassium $\left(156.4 \mathrm{~kg} \mathrm{ha}^{-1}\right)$ was recorded in treatment $\mathrm{T}_{4}$ (Seasoned pressmud @ $25 \mathrm{tha}^{-1}+$ lignite fly ash @ $25 \mathrm{tha}^{-1}$ ).

Key Words : Seasoned pressmud, Enriched gypsum, $\mathrm{ZnSO}_{4}$, Lignite fly ash, Yield

How to cite this article : Venkatakrishnan, D. (2016). Response of manures and industrial by-products for cane yield and post harvest NPK status . Internat. J. Plant Sci., 11 (2): 359-363, DOI: 10.15740/HAS/IJPS/11.2/359-363.

Article chronicle : Received : 05.02.2016; Revised : 01.06.2016; Accepted : 28.06.2016

\section{AUTHOR FOR CORRESPONDENCE}

D. VENKATAKRISHNAN, Department of Soil Science and Agricultural Chemistry, Annamalai University, Annamalainagar, CHIDAMBARAM (T.N.) INDIA

Email: mahemasree@yahoo.co.in 